\title{
Stable reversal of multidrug resistance in colon cancer cells by RNA interference targeting the MDR1 gene
}

\author{
ZHONGSHENG XIA ${ }^{1}$, LIYONG ZHANG ${ }^{2}$, QIKUI CHEN ${ }^{1}$, CRISTA ROYAL $^{3}$, \\ ZHONG YU $^{1}$, ZHONGMIN LIU ${ }^{2}$ and BAO-LING ADAM ${ }^{2}$
}

\begin{abstract}
${ }^{1}$ Department of Gastroenterology, The Second Affiliated Hospital, Sun Yat-sen University, Guangzhou 510120, P.R. China;
${ }^{2}$ Center for Biotechnology and Genome Medicine, and ${ }^{3}$ Department of Pharmacology and Toxicology,

Medical College of Georgia, Augusta, GA 30912, USA
\end{abstract}

Received January 2, 2009; Accepted March 24, 2009

DOI: $10.3892 / \mathrm{mmr}+00000140$

\begin{abstract}
Colon cancer is one of the most common cancers in the world. Overexpression of MDR1 mRNA and P-gp is associated with the classic multidrug resistance of colon cancer cells. In our previous study, we reported on the transient specific reversal of MDR1/P-gp-dependent multidrug resistance by RNA interference (RNAi) in colon cancer cells. In this study, RNAi targeting the MDR1 gene stably reversed MDR1/P-gp-dependent multidrug resistance in colon cancer cells. The plasmid vectors pSilencer-\#4029, encoding \#4029 MDR1 siRNA, and pSilencer-\#4123, encoding \#4123 MDR1 siRNA, were constructed and then transfected into COLO 320DM, a colon cancer multidrug-resistant cell line. Clone cells were screened by G418 and identified by RT-PCR and Western blot analysis. Cellular viability was measured using the MTT assay. Cell cycle analysis and intracellular adriamycin accumulation were assessed by flow cytometry. MDR1 mRNA and P-gp expression in positive clone cells, those stably transfected with MDR1 siRNA, was inhibited. The $\mathrm{IC}_{50}$ values of the antitumor drugs were significantly decreased in the positive clones compared to the COLO 320DM parent cell line, and the PI/AI values of positive clones treated with antitumor drugs were significantly decreased compared to the parent cells. In addition, the intracellular adriamycin accumulation of positive clones treated with adriamycin was significantly increased compared with COLO 320DM. This demonstrates that the stable transfection of plasmid vectors encoding MDR1 siRNA can stably reverse the MDR1/P-gp-dependent multidrug resistance of colon cancer cells.
\end{abstract}

Correspondence to: Dr Zhongsheng Xia, Department of Gastroenterology, The Second Affiliated Hospital, Sun Yat-sen University, Guangzhou 510120, P.R. China

E-mail: xiazhsh@hotmail.com

Key words: RNA interference, stable transfection, colon cancer, multidrug resistance, MDR1, P-glycoprotein

\section{Introduction}

Colon cancer is the second leading cause of cancer-related death in the US (1). This may in part be due to the fact that in most patients the disease has reached an advanced stage by the time of diagnosis (2). Chemotherapy plays an important role in the treatment of colon cancer. However, in colon cancer cells the effectiveness of chemotherapy is limited due to multidrug resistance (MDR) (3).

One of the underlying mechanisms conferring the MDR phenotype is overexpression of the MDR 1 gene encoding a 170-kDa transmembrane P-glycoprotein (MDR1/P-gp) (4), a member of the superfamily of ABC (adenosine triphosphate binding cassette)-transporters (5). P-glycoprotein (P-gp) functions as an energy-dependent efflux pump, resulting in decreased concentrations of intracellular drugs and resistance to their chemotherapeutic effect. In previous studies, calcium antagonists such as verapamil (6), antisense oligonucleotides (7) and hammerhead ribozymes (8) were found to modulate MDR1/P-gp-dependent MDR by down-regulating the expression of MDR1 mRNA and P-gp. However, calcium antagonists may cause heart failure and hypotension (9), and the clinical benefit of antisense oligonucleotides and hammerhead ribozymes has not been realized. Consequently, it is necessary to develop alternative, less toxic and more efficient strategies or pharmaceuticals to reverse the MDR of cancer cells.

It has been shown that RNA interference (RNAi) can knock down gene expression. In our previous study, siRNAs targeting MDR1 mRNA in colon cancer cells suppressed its expression as well as that of $\mathrm{P}$-gp, reversing the MDR of colon cancer cells (10). However, a major disadvantage of this approach is the transient gene expression-inhibiting effect of chemically synthesized siRNAs. Therefore, the development of more effective and stable gene-silencing RNAi-mediating systems is of great interest.

In this study, we constructed two siRNA-expressing plasmid vectors (pSilencer-\#4029 MDR1 and pSilencer-\#4123 MDR1), which orchestrated the synthesis of siRNAs directed against the MDR1/P-gp-specific transcript. We then screened cells stably transfected with siRNA expression vectors for positively-transfected clones. Finally, representative clones were selected and further characterized, including an examination of the gene silencing effects of the siRNA, in particular 
MDR1 mRNA and P-gp expression, $\mathrm{IC}_{50}$ determination, cell kinetics analysis and antitumor drug intracellular accumulation.

\section{Materials and methods}

Materials. The plasmid pSilencer ${ }^{\mathrm{TM}}$ 2.1-U6 neo vector kit was purchased from Ambion Co. (Austin, TX). Competent bacteria DH5 $\alpha^{\mathrm{TM}}$ E. coli, S.O.C. medium, Opti-MEM ${ }^{\circledR}$ I reduced serum medium, liposome Lipofectamine ${ }^{\mathrm{TM}}$ 2000, TRIzol reagent and a cDNA synthesis kit were purchased from Invitrogen Co. (Carlsbad, CA). The Wizard Plasmid DNA Purification kit was purchased from Promega Co. (Madison, WI). The COLO 320DM human colon adenocarcinoma cell line was purchased from ATCC (American Type Culture Collection, Manassas, VA) and cultured in RPMI-1640 supplemented with 10\% FBS (Sigma, St. Louis, MO), $100 \mathrm{U} / \mathrm{ml}$ penicillin (Mediatech, Inc., Herndon, VA) and $100 \mu \mathrm{g} / \mathrm{ml}$ streptomycin (Mediatech, Inc.) at $37^{\circ} \mathrm{C}$ in a humidified incubator containing $5 \% \mathrm{CO}_{2}$. Adriamycin, vincristine, ampicilline, G418 and mouse antihuman $\beta$-actin $\mathrm{mAb}$ were purchased from Sigma. RPMI1640 medium was purchased from Hyclone Co. (Waltham, MA). C219 (mouse anti-human P-gp mAb) was purchased from Signet Co. (El Monte, CA). The ECL chemiluminescence system was purchased from Amersham Bioscience Co. (Piscataway, NJ).

Construction of siRNA expression vectors. Two siRNA sequences targeting the MDR1 coding regions 1786-1804 (5'-GGATATTAGGACCATAAAT-3', GenBank accession no. NM_000927) and 665-683 (5'-GGAAATTTAGAAG ATCTGA-3', GenBank accession no. NM_000927) were selected based on the results of transient transfection (10). Two oligonucleotides encoding small hairpin RNA (shRNA) containing sense and antisense sequences linked by a hairpin loop (TTCAAGAGA) were synthesized. The sequence of the \#4029 MDR1 oligonucleotide was as follows: sense strand, 5'-GAT CCG GAA ATT TAG AAG ATC TGA TTC AAG AGA TCA GAT CTT CTA AAT TTC CTG TTT TTT GGA AA-3'; antisense strand, 5'-AGC TTT TCC AAA AAA CAG GAA ATT TAG AAG ATC TGA TCT CTT GAA TCA GAT CTT CTA AAT TTC CG-3'. The sequence of the \#4123 MDR1 oligonucleotide was as follows: sense strand, 5'-GAT CCG GAT ATT AGG ACC ATA AAT TTC AAG AGA ATT TAT GGT CCT AAT ATC CTG TTT TTT GGA AA-3'; antisense strand, 5'-AGC TTT TCC AAA AAA CAG GAT $\underline{\text { ATT AGG ACC ATA AAT TCT CTT GAA ATT TAT GGT }}$ CCT AAT ATC CG-3'. These oligonucleotides were annealed in DNA annealing solution at $90^{\circ} \mathrm{C}$ for $3 \mathrm{~min}$ and $37^{\circ} \mathrm{C}$ for $1 \mathrm{~h}$. The two annealed inserts encoding shRNA were ligated with linear pSilencer 2.1-U6 neo vector containing the neomycin resistance gene, Bam $\mathrm{H} 1$ and HindIII restriction sites. The negative control vector contained no sequence homologous to any known human genes. Plasmid DNA was extracted from E. coli DH5 $\alpha$ transformants using the Promega Wizard Genomic DNA Purification kit. The two MDR1 siRNA constructs (pSilencer-\#4029 MDR1 and pSilencer-\#4123 MDR1) were confirmed by DNA sequencing.

Transfection and selection of stable transfected clones. The COLO 320DM cell line was transfected with $2 \mu \mathrm{g}$ of expres- sion vector DNA, pSilencer-\#4029 MDR1, pSilencer-\#4123 MDR1, and negative control vector. Each experiment was performed in six $30-50 \%$ confluent wells using $10 \mu \mathrm{l}$ of Lipofectamine 2000 transfection reagent. The transfected cells were selected in G418 $(400 \mu \mathrm{g} / \mathrm{ml})$ containing cell culture medium. After 3 weeks, visible clones were selected in 96 wells, expanded in 24 and 6 wells, and transferred to regular cell culture flasks. Various pSilencer-\#4029 MDR1, pSilencer\#4123 MDR1 and negative control vector-transfected clones derived from COLO 320DM cells were isolated and expanded. Gene silencing activity in the clones was verified using RT-PCR and Western blot analysis. The gene silencing effects of the siRNA were characterized using representative clones. All representative clones were frozen in liquid nitrogen for one month, then resuscitated, cultured, and passaged for another month. Finally, gene silencing activity was again verified by RT-PCR and Western blotting.

Real-time RT-PCR. Total RNA was isolated from cells using TRIzol reagent according to the manufacturer's instructions. Single-stranded cDNA was synthesized from $1 \mu \mathrm{g}$ RNA using a cDNA synthesis kit. The newly synthesized cDNA was amplified by PCR using an iCycle instrument (Bio-Rad, Hercules, CA). The reaction mixture contained $1 \mu \mathrm{l}$ of cDNA template, $1.5 \mathrm{mM} \mathrm{MgCl}_{2}, 2.5$ units Taq polymerase, 10 pmol MDR1 sense primer and 10 pmol MDR1 antisense primer, $0.63 \mu 1 \mathrm{SYBR}$ green I and $0.25 \mu 1 \mathrm{FCD}$ (Roche, Florence, SC). The final reaction volume was $25 \mu \mathrm{l}$. Both sense and antisense $B$-actin primers were used as internal controls. Cycling conditions were as follows: $95^{\circ} \mathrm{C}$ for 5 min for cDNA and primer denaturing, followed by 40 cycles at $95^{\circ} \mathrm{C}$ for $30 \mathrm{sec}$, $55^{\circ} \mathrm{C}$ (MDR 1$)$ or $56^{\circ} \mathrm{C}$ ( $ß$-actin) for $30 \mathrm{sec}$, and $72^{\circ} \mathrm{C}$ for $30 \mathrm{sec}$. Based on the fluorescence intensity curve, the appropriate amplification cycles ( 26 cycles for MDR 1 and 23 cycles for $\beta$-actin, with a final cycle at $72^{\circ} \mathrm{C}$ for $5 \mathrm{~min}$ ) were chosen. Aliquots of PCR product were electrophoresed using $1.5 \%$ agarose gels, and PCR fragments were visualized by ethidium bromide staining. The amplification products of MDR1 and $ß$-actin were 161 and $254 \mathrm{bp}$, respectively.

Western blot analysis. Cells were washed twice with PBS and lysed with lysis buffer (30 mM Tris- $\mathrm{HCl} \mathrm{pH} 7.4,1 \%$ SDS), then the lysates were centrifuged at $12,000 \times \mathrm{g}$ at $4^{\circ} \mathrm{C}$ for 15 min. Total cellular protein was determined using the $\mathrm{BCA}^{\mathrm{TM}}$ protein assay kit (Pierce, Rockford, IL). Subsequently, cellular proteins were run on 7.5\% SDS-PAGE gels and electrotransferred onto PVDF membranes (100 V, $2.5 \mathrm{~h})$. The membranes were rinsed with PBS and blocked with $10 \%$ nonfat milk in PBS for $3 \mathrm{~h}$ at room temperature, then incubated with the C219 (1:100) (Signet) or $\beta$-actin $(1: 4000)$ (Sigma) primary antibody in $3 \%$ nonfat milk. Following incubation with the antibodies, the membranes were rinsed in TBS-T wash buffer for 5 min 6 times each, then incubated with secondary antibody (HRP-conjugated goat anti-mouse $\mathrm{IgG}, 1: 6000$ ) (Pierce) for $1 \mathrm{~h}$ at room temperature. After rinsing, the protein-antibody complexes were visualized using the ECL chemiluminescence system.

$I C_{50}$ determination of antitumor drugs. To determine the $\mathrm{IC}_{50}$ of antitumor drugs in the COLO 320DM parent cell line and clone 
A

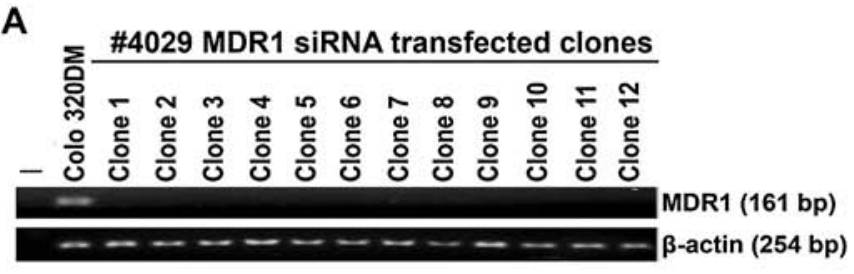

B

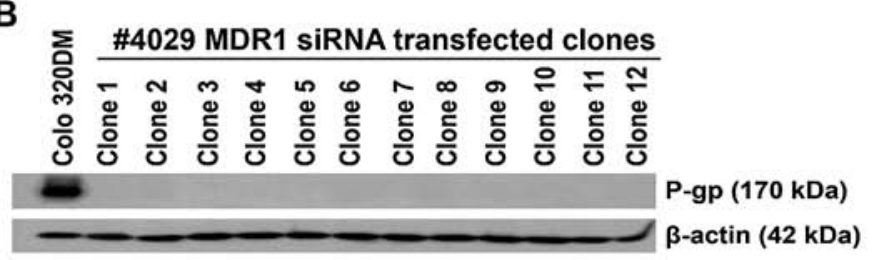

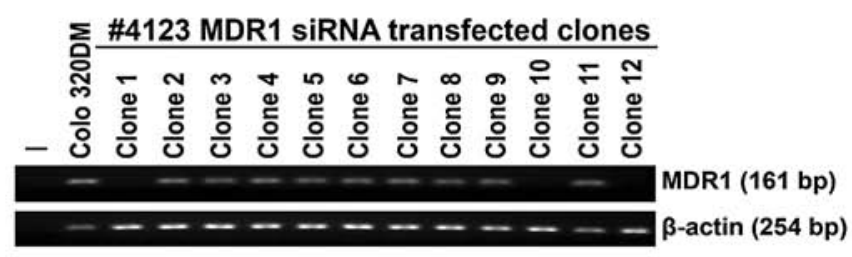

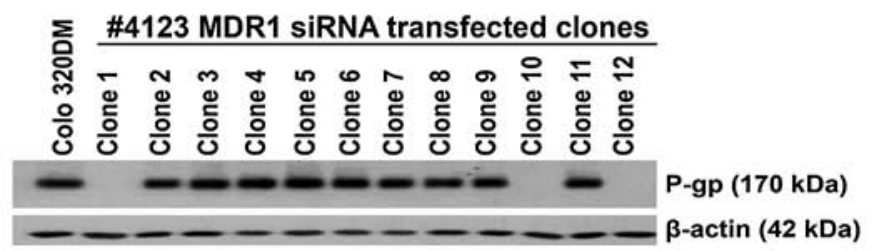

Figure 1. (A) RT-PCR analysis of MDR1 mRNA expression in the COLO 320DM parent cell line and clones stably transfected with siRNA. -, no cDNA template added. (B) Western blot analysis of P-gp expression in the COLO 320DM parent cell line and clones stably transfected with siRNA.

cells, different concentrations of adriamycin (0-20.48 $\mu \mathrm{mol} / \mathrm{l})$ and vincristine $(0-6.5536 \mu \mathrm{mol} / \mathrm{l})$ were applied for 3 days in a series of 2-fold dilutions to treat the COLO 320DM parent cell line and clone cells. Cell viability was measured using the MTT assay (11). $\mathrm{IC}_{50}$ values were calculated.

Cell kinetics analysis. COLO 320DM parent and clone cells were plated at $5 \times 10^{5}$ cells/well in 6 -well plates in RPMI-1640 medium with $10 \%$ FBS. After 6 h, antitumor drugs including adriamycin and vincristine at a final concentration of 0.32 and $0.0128 \mu \mathrm{mol} / 1$, respectively, were added. The cells were incubated at $37^{\circ} \mathrm{C}$ in $5 \% \mathrm{CO}_{2}$, harvested after $72 \mathrm{~h}$, washed twice with cold PBS, then fixed with $70 \%(\mathrm{v} / \mathrm{v})$ ethanol at $4^{\circ} \mathrm{C}$ for at least $1 \mathrm{~h}$. After fixation, the cells were washed twice with cold PBS. Cellular DNA was stained with propidium iodide staining solution $(3.8 \mathrm{mM}$ sodium citrate, $50 \mu \mathrm{g} / \mathrm{ml}$ propidium iodide) and $20 \mu \mathrm{g} / \mathrm{ml}$ RNase A for $30 \mathrm{~min}$ at room temperature. Flow cytometry analysis was performed using the FACSCalibur $^{\text {TM }}$ flow cytometer (Becton Dickinson, San Jose, CA). In each sample, 10,000 cells were analyzed. Cell cycle analysis was performed using the BD CellQuest ${ }^{\text {TM }}$ PRO (version 5.2.1) software package (Becton Dickinson). The percentage of each cell cycle phase was calculated using the formula: proliferation index $(\mathrm{PI})=\left(\mathrm{S}+\mathrm{G}_{2} / \mathrm{M}\right) /\left(\mathrm{G}_{0} / \mathrm{G}_{1}+\mathrm{S}+\mathrm{G}_{2} / \mathrm{M}\right)$ $\mathrm{x} 100 \%$; apoptosis index $(\mathrm{AI})=\left(\mathrm{G}_{0} / \mathrm{G}_{1}\right) /\left(\mathrm{G}_{0} / \mathrm{G}_{1}+\mathrm{S}+\mathrm{G}_{2} / \mathrm{M}\right) \mathrm{x}$ $100 \%$. PI/AI represents the change in cell kinetics (12).

Intracellular adriamycin accumulation assay. The fluorescence intensity of intracellular adriamycin was determined by flow cytometry according to standard procedures (13). The cells were plated at a density of $2 \times 10^{5}$ cells/well in 6-well plates and incubated for $48 \mathrm{~h}$. Subsequently, adriamycin at a final concentration of $10 \mu \mathrm{mol} / \mathrm{l}$ was added, and the cells were incubated for $2 \mathrm{~h}$, harvested, washed twice with cold PBS, and then placed in ice-water to block the reaction until analysis. After half an hour, the fluorescence intensity of cells was determined using the FACSCalibur flow cytometer at an excitation wavelength of $488 \mathrm{~nm}$ and a receiving wavelength of $575 \mathrm{~nm}$.

\section{Results}

Expression of MDRI $\mathrm{mRNA}$ and P-gp in the COLO 320DM parent cell line and stable transfected clones. Clones derived from the COLO 320DM parent cell line transfected with plasmid vector encoding \#4029 MDR1 siRNA, \#4123 MDR1 siRNA and negative control vector were identified by RT-PCR and Western blot analysis. Positive clones whose MDR1 RNA and P-gp were stably suppressed were selected for further study. As shown in Fig. 1A, \#4029 MDR1 siRNA was stably expressed by all the clones (nos. 1-12), indicating MDR 1 mRNA expression was inhibited in these clones. Western blot analysis confirmed the results of RT-PCR analysis (Fig. 1B). Clones 4, 5, and 6 were selected for further analysis. Data from these three clones stably expressing \#4029 MDR1 siRNA were merged into the labeled clone \#4029. For the \#4123 MDR1 siRNA sequence, the clones 1, 10 and 12 stably expressed \#4123 MDR1 siRNA, indicating MDR1 mRNA expression was suppressed in these clones (Fig. 1A). Western blot analysis confirmed the results of RT-PCR (Fig. 1B). Clones 1, 10 and 12 were selected for further analysis. Data from these three clones stably expressing \#4123 MDR1 siRNA were merged into the labeled clone \#4123.

$I C_{50}$ of antitumor drugs in the COLO 320DM parent cell line and stably transfected siRNA clones. The $\mathrm{IC}_{50}$ values of adriamycin and vincristine were significantly decreased in the clones stably expressing \#4029 MDR1 siRNA or \#4123 MDR1 siRNA compared with the COLO 320DM parent cells. However, no significant differences in $\mathrm{IC}_{50}$ values were observed between the negative control and parent cells (Table I). Among the clones stably expressing \#4029 MDR1 siRNA, the $\mathrm{IC}_{50}$ of adriamycin was decreased $~ 9$-fold (9.616$1.094 \mu \mathrm{mol} / 1)$. For the \#4123 MDR1 siRNA-expressing clones, the $\mathrm{IC}_{50}$ of adriamycin was decreased $\sim 12$-fold (9.616-0.780 $\mu \mathrm{mol} / 1$. The $\mathrm{IC}_{50}$ of vincristine for clone \#4029 was decreased $\sim 15$-fold $(0.358-0.023 \mu \mathrm{mol} / 1)$, while in clone \#4123 it was decreased $\sim 10$-fold $(0.358-0.035 \mu \mathrm{mol} / \mathrm{l})$. 
Table I. $\mathrm{IC}_{50}$ of adriamycin and vincristine in the COLO 320DM colon cancer parent cell line and its derivative clone cells.

\begin{tabular}{lcc}
\hline Group & $\begin{array}{c}\text { Adriamycin } \\
(\mu \mathrm{mol} / \mathrm{l})\end{array}$ & $\begin{array}{c}\text { Vincristine } \\
(\mu \mathrm{mol} / \mathrm{l})\end{array}$ \\
\hline Parent & $9.616 \pm 0.115$ & $0.358 \pm 0.010$ \\
Negative control & $8.279 \pm 0.100$ & $0.435 \pm 0.011$ \\
Clone \#4029 & $1.094 \pm 0.141^{\mathrm{a}}$ & $0.023 \pm 0.010^{\mathrm{a}}$ \\
Clone \#4123 & $0.780 \pm 0.121^{\mathrm{a}}$ & $0.035 \pm 0.012^{\mathrm{a}}$ \\
\hline
\end{tabular}

a $<<0.01$ compared with parent.

Table II. PI/AI of the COLO 320DM parent cell line and its derivative clone cells treated with adriamycin and vincristine.

\begin{tabular}{lc}
\hline Group & PI/AI \\
\hline Parent & $12.54 \pm 5.39$ \\
Negative control & $17.74 \pm 9.46$ \\
Clone \#4029 & $13.27 \pm 5.87$ \\
Clone \#4123 & $12.19 \pm 9.58$ \\
Parent-adriamycin & $5.68 \pm 0.19$ \\
Negative control-adriamycin & $7.09 \pm 1.86$ \\
Clone \#4029-adriamycin & $2.74 \pm 0.45^{\mathrm{a}}$ \\
Clone \#4123-adriamycin & $2.75 \pm 0.36^{\mathrm{a}}$ \\
Parent-vincristine & $9.59 \pm 1.00$ \\
Negative control-vincristine & $6.90 \pm 0.94$ \\
Clone \#4029-vincristine & $3.59 \pm 0.28^{\mathrm{b}}$ \\
Clone \#4123-vincristine & $3.24 \pm 0.35^{\mathrm{b}}$ \\
\hline
\end{tabular}

${ }^{\text {aP }}<0.01$ compared with parent-adriamycin. ${ }^{\text {b }}<0.01$ compared with parent-vincristine. PI, proliferation index; AI, apoptosis index.

Cell kinetics analysis of adriamycin-and vincristine-treated COLO 320DM parent and clone cells. In order to investigate the changes in cell kinetics of COLO 320DM parent and clone cells treated with antitumor drugs, cell cycle analysis was performed with flow cytometry. As shown in Table II, there was no difference in PI/AI among COLO 320DM parent and clone cells before treatment with antitumor drugs. Compared with pre-treatment values, the PI/AI significantly decreased in all groups after treatment with antitumor drugs. Furthermore, the PI/AI of clone \#4029 and clone \#4123 decreased to 2.74 and 2.75 , respectively, from 5.68 of COLO 320DM parent cells after treatment with adriamycin. Vincristine treatment also decreased the PI/AI of clone \#4029 and clone \#4123 to 3.59 and 3.24, respectively, from 9.59 of COLO 320DM parent cells.

Intracellular adriamycin accumulation in COLO 320DM parent and clone cells. An intracellular adriamycin accumulation assay demonstrated that there was no significant
Table III. Fluorescence intensity in the COLO 320DM parent cell line and its derivative clone cells.

\begin{tabular}{lc}
\hline Group & Fluorescence intensity \\
\hline Parent & $4.58 \pm 0.17$ \\
Negative control & $6.48 \pm 0.46$ \\
Clone \#4029 & $6.58 \pm 0.20$ \\
Clone \#4123 & $7.50 \pm 0.46$ \\
Parent-adriamycin & $27.92 \pm 0.39^{\mathrm{a}}$ \\
Negative control-adriamycin & $36.84 \pm 3.38^{\mathrm{a}}$ \\
Clone \#4029-adriamycin & $187.24 \pm 5.93^{\mathrm{ab}}$ \\
Clone \#4123-adriamycin & $215.57 \pm 20.31^{\mathrm{ab}}$ \\
\hline
\end{tabular}

${ }^{a} \mathrm{P}<0.01$ compared with parent. ${ }^{b} \mathrm{P}<0.01$ compared with parentadriamycin.

difference in intracellular adriamycin accumulation between the COLO 320DM parent cell line (control or parent) and each clone derived from the COLO 320DM parent cell line. Moreover, the fluorescence intensity of adriamycin, a measure of intracellular adriamycin accumulation, was 27.92 in the COLO 320DM parent cell line after adriamycin treatment, with no significant difference between the parent and negative control clone cells (Table III). However, intracellular adriamycin accumulation was significantly higher in each clone stably expressing \#4029 MDR1 siRNA and \#4123 MDR1 siRNA compared to the parent cells. In clone \#4029, its accumulation was increased to 187.24 (5.71-fold), while in clone \#4123 it was increased to 215.57 (6.72-fold).

\section{Discussion}

Multidrug resistance (MDR) may be due to the overexpression of the ATP-binding cassette. Classic MDR (MDR1/P-gpdependent MDR) is caused by the overexpression of $\mathrm{P}$-glycoprotein (P-gp) in cancer cells. If MDR is related to the overexpression of other proteins, such as multidrug resistanceassociated protein (MRP) or breast cancer resistance protein (BRCP), it is designated atypical or non-MDR1/P-gpdependent MDR.

COLO 320DM is a multidrug-resistant human colon cancer cell line that is resistant to many antitumor drugs, including adriamycin and vincristine. COLO 320DM expresses P-gp, but not MRP (14), and is therefore an MDR1/P-gp-dependent multidrug-resistant colon cancer cell line. In contrast, HT-29 is a non-MDR1/P-gp-dependent multidrug-resistant human colon cancer cell line. HT-29 does not express P-gp (14), but does exhibit MDR related to the expression of MRP (15). In our previous study (10), the human COLO 320DM colon cancer cell line with HT-29 cells as the control were used to investigate the reversal of MDR1/P-gp-dependent MDR by \#4123 and \#4029 MDR1 siRNAs targeting MDR1 mRNA. The efficacy of the MDR1 siRNAs in knocking down the phenotype of MDR in COLO 320DM cells, without knocking down MDR effects in the HT-29 control cells, was clearly demonstrated. Furthermore, MDR1 siRNAs enhanced the 
cytotoxicity of the antitumor drugs adriamycin and vincristine in the COLO 320DM cells, but not in the HT-29 control cell line. This revealed the specificity of MDR1 siRNA targeting to MDR1 mRNA. Based on these findings regarding the specific effects of MDR1 siRNAs (10), in the present study only the COLO 320DM cell line was employed to investigate the stable reversal of colon cancer cell MDR. Other studies have reported the stable reversal of MDR in colon cancer cells (16). However, the colon cancer cell lines employed in these studies were far from optimal, as the MDR in these colon cell lines was related to the overexpression of MRP and BCRP in addition to the overexpression of MDR1. In contrast, the COLO 320DM cell line only overexpresses MDR1. It is a classic multidrug-resistant colon cancer cell line; i.e., a MDR1/P-gp-dependent multidrug-resistant colon cancer cell line. Therefore, we strongly believe that the COLO 320DM cell line is preferable for investigating the stable reversal effect of MDR1/P-gp-dependent MDR in colon cancer cells.

It is known that not all positive synthetic siRNA oligonucleotides work in vector-based RNAi; therefore two different oligonucleotide hairpin sequences (the \#4029 MDR1 and \#4123 MDR1 oligonucleotides) were designed, cloned into the pSilencer 2.1-U6 neo vectors, and used to generate stable RNAi clones. The pSilencer 2.1-U6 neo vector contains neomycin resistance, allowing only for the selection of clones containing the hairpin sequence.

One major disadvantage of transient transfection is the transient gene expression-inhibiting effect. RNAi effects usually last only a few days (17). Our previous study also confirmed this transient effect (10). MDR1 mRNA and P-gp expression were suppressed for 4, 5 and 6 days when the COLO 320DM colon cancer cells were transfected with 5, 25 and $100 \mathrm{nmol} / \mathrm{l}$, respectively, of \#4123 MDR1 siRNA. The maximum effect duration was therefore only 6 days. To achieve longer and more stable inhibition of MDR1 mRNA and P-gp expression in COLO 320DM cells, in this study stable transfections were performed after the sequences of effective MDR1 siRNA were found in the transient transfection experiments. The COLO 320DM cells transfected with siRNA expression vector were screened for positive stably transfected clones using G418.The positive clones were verified by RTPCR and Western blotting, then cultured and passaged for over a month, frozen in liquid nitrogen for a month, and lastly resuscitated, cultured, and passaged for another month. They were then tested once again for gene silencing activity by RT-PCR and Western blotting. MDR1 mRNA and P-gp were found to be stably suppressed in all the positive clones. This indicates that MDR1 siRNA expression plasmid vectors stably inhibited the expression of MDR1 mRNA and P-gp in the COLO 320DM colon cancer multidrug-resistant cell line. Further characterization of the gene silencing effects of the siRNA, including $\mathrm{IC}_{50}$ determination, cell kinetics analysis and intracellular antitumor drug accumulation, was carried out in representative clones.

The MTT assay was used to measure the $\mathrm{IC}_{50}$ of adriamycin and vincristine in clones derived from the multidrug-resistant COLO 320DM parent cell line, which were transfected with \#4029 MDR1 siRNA- or \#4123 MDR1 siRNA-expressing plasmid vector. Expression of MDR1 mRNA and P-gp was inhibited in the clones, and $\mathrm{IC}_{50}$ values were decreased com- pared with the parent cells. This suggests that clone cells transfected with the MDR1 siRNA expression vector were more sensitive to the antitumor drugs adriamycin and vincristine than the COLO 320DM parent cell line, consistent with the results of transient transfection (10). This conclusion was confirmed by intracellular adriamycin accumulation analysis, which revealed that intracellular adriamycin accumulation was increased more than 5-fold after treatment with adriamycin in the positive clones vs. the multidrug-resistant COLO 320DM parent cell line. This indirectly indicates that the pump function of P-gp in positive clone cells was suppressed. Cell kinetics analysis demonstrated that the PI/AI values of positive closnes were significantly decreased compared to the parent cells after treatment with the antitumor drugs adriamycin and vincristine. Collectively, the above data demonstrate that positive clone cells transfected with MDR1 siRNA-expressing vector can stably reverse the phenotype of MDR1/P-gpdependent MDR in colon cancer cells.

However, the inhibiting effects of MDR 1 siRNA on MDR1 mRNA and P-gp in the COLO 320DM cell line were obtained in vitro. Such inhibiting effects need to be investigated in vivo in future studies. In addition, a problem in clinical application needs to be considered: MDR1 gene products are present not only in malignant cells, but also in various normal tissues, such as the peripheral blood cells and hemopoietic progenitors found in normal human bone marrow (18). Although MDR1 expression in these tissues is relatively low, P-gp expression may be functionally important in protecting these rapidly dividing cells from toxicity after exposure to anticancer drugs (19). Thus, in a clinical context, it may be necessary for the application of the MDR1 siRNA expression vector to be restricted to MDR1/P-gp-expressing multidrug-resistant cancer cells only. One promising strategy for cancer cell-limited delivery of MDR1 siRNA is the effort to develop vector systems specific to multidrug-resistant cancer cells. Recently, a 'replication-defective' E1A-mutant adenoviral vector, which efficiently and selectively replicates in 'classic' multidrug-resistant cells, was described (20). This type of virus may provide the basis for the development of novel vectors for the specific gene therapeutic treatment of multidrug-resistant cancer cells. Though such a specific delivery system has yet to be developed, we are encouraged by the discovery that RNAi targeting the MDR1 gene can stably reverse MDR1/P-gp-dependent MDR and increase antitumor drug sensitivity in colon cancer cells.

\section{References}

1. Hawk ET, Limburg PJ and Viner JL: Epidemiology and prevention of colorectal cancer. Surg Clin North Am 82: 905-941, 2002.

2. Taa BG, Van Tinteren $\mathrm{H}$ and Zoetmulder FA: Adjuvant 5-FU plus levamisole in colonic or rectal cancer: improved survival in stage II and III. Br J Cancer 85: 1437-1443, 2001.

3. Redmond SM, Joncourt F, Buser K, et al: Assessment of P-glycoprotein, glutathione-based detoxifying enzymes and O6alkyl-guanine-DNA alkyltransferase as potential indicators of constitutive drug resistance in human colorectal tumors. Cancer Res 51: 2092-2097, 2001.

4. Germann UA, Pastan I and Gottesman MM: P-glycoproteins: mediators of multidrug resistance. Semin Cell Biol 4: 63-76, 1993.

5. Lage $\mathrm{H}$ : ABC-transporters: implication on drug resistance from microorganisms to human cancers. Int J Antimicrob Agents 22: 188-199, 2003. 
6. Van de Vrie W, Gheuens EE, Durante NM, et al: In vitro and in vivo chemosensitizing effect of cyclosporin $\mathrm{A}$ on an intrinsic multidrug-resistant rat colon tumour. J Cancer Res Clin Oncol 119: 609-614, 1993.

7. Quattrone A, Papucci L, Morganti M, et al: Inhibition of MDR1 gene expression by antimessenger oligonucleotides lowers multiple drug resistance. Oncol Res 6: 311-320, 1994.

8. Kobayashi H, Dorai T, Holland JF, et al: Reversal of drug sensitivity in multidrug-resistant tumor cells by an MDR1 (PGY1) ribozyme. Cancer Res 54: 1271-1275, 1994.

9. Damiani D, Michieli M, Michelutti A, et al: D-verapamil downmodulates P170-associated resistance to doxorubicin, daunorubicin and idarubicin. Anticancer Drugs 4: 173-180, 1993.

10. Xia Z, Zhu Z, Zhang L, et al: Specific reversal of MDR1/P-gpdependent multidrug resistance by RNA interference in colon cancer cells. Oncol Rep 20: 1433-1439, 2008.

11. Carmichael J, Degraff WG, Gazdar AF, et al: Evaluation of a tetrazolium-based semiautomated colorimetric assay: assessment of chemosensitivity testing. Cancer Res 47: 936-942, 1987.

12. Bai M, Agnantis NJ, Kamina S, et al: In vivo cell kinetics in breast carcinogenesis. Breast Cancer Res 3: 276-283, 2001.

13. Li J, Xu LZ, He KL, et al: Reversal effects of nomegestrol acetate on multidrug resistance in adriamycin-resistant MCF7 breast cancer cell line. Breast Cancer Res 3: 253-263, 2001
14. Van Rensburg CE, Joone GK and O'Sullivan JF: Clofazimine and B4121 sensitize an intrinsically resistant human colon cancer cell line to P-glycoprotein substrates. Oncol Rep 7: 193-195, 2000.

15. Kok JW, Veldman RJ, Klappe K, et al: Differential expression of sphingolipids in MRP1 overexpressing HT29 cells. Int J Cancer 87: 172-178, 2000.

16. Celius T, Garberg P and Lundgren B: Stable suppression of MDR1 gene expression and function by RNAi in Caco- 2 cells. Biochem Biophys Res Commun 324: 365-371, 2004.

17. Nieth C, Priebsch A, Stege A, et al: Modulation of the classical multidrug resistance (MDR) phenotype by RNA interference (RNAi). FEBS Lett 545: 144-150, 2003.

18. Licht T, Pastan I, Gottesman M, et al: P-glycoprotein-mediated multidrug resistance in normal and neoplastic hematopoietic cells. Ann Hematol 69: 159-171, 1994.

19. Smeets M, Raymakers R, Vierwinden G, et al: A low but functionally significant MDR1 expression protects primitive haemopoietic progenitor cells from anthracycline toxicity. Br J Haematol 96: 346-355, 1997.

20. Holm PS, Lage H, Bergmann S, et al: Multidrug-resistant cancer cells facilitate E1-independent adenoviral replication: impact for cancer gene therapy. Cancer Res 64: 322-328, 2004. 